\title{
Application of genomic evaluation to dairy cattle in New Zealand
}

\author{
A. M. Winkelman, ${ }^{1}$ D. L. Johnson, and B. L. Harris \\ LIC, Private Bag 3016, Hamilton, 3240, New Zealand
}

\begin{abstract}
A hybrid method of estimating genomically enhanced breeding values (GEBV) that uses features of a single-step approach and traditional national estimated breeding value (EBV), based on pedigree, as the starting point was developed and assessed. The method uses deregressed EBV from the traditional EBV estimation procedures and a pedigree matrix that is augmented by a genomic relationship matrix based on a Euclidean distance (EDM) in a Gaussian kernel. Genotypes were obtained using the Illumina BovineSNP50 BeadChip (Illumina Inc., San Diego, CA). The pedigree information is restricted to animals with genotypes, as well as all of their ancestors, resulting in a system of equations that is considerably smaller than that of the national evaluation. Genome-wide association studies were used to identify regions of the genome that had high association with the traits in the New Zealand national breeding objective. The EDM were created for each selected region. The amount of variance accounted for by each region was estimated, and the variances, relative to the EDM excluding these areas, were used to create traitspecific weighted EDM that were used for the evaluations. The evaluation is done on the restricted data set and is a reversal of the deregression procedure using the augmented pedigree-based relationship matrix. The method was tested using data on 7,204 progeny-tested sires, in which the daughter records for the 3 youngest cohorts (i.e., validation data; $\mathrm{n}=1,410$ ) were excluded. Evaluations were done for the 7 traits (3 production, 4 nonproduction) in the New Zealand national breeding objective. Regression of the traditional EBV based on daughter information on the GEBV for validation sires had an average slope of 0.99 (range 0.93 to 1.06); the average correlation between the EBV was 0.79 (range 0.56 to 0.84 ). The average slope and correlation for the previously used blended GEBV were 0.64 and 0.61 , respectively. The method was applied to all genotyped animals that included approximately 13,000 genotyped males and 47,000 genotyped females and their ances-
\end{abstract}

Received July 2, 2014.

Accepted September 15, 2014.

${ }^{1}$ Corresponding author: awinkelman@lic.co.nz tors. The correlation between the traditional EBV and GEBV of the proven bulls was 0.99; the correlations obtained from the previously used blended GEBV were 0.88 (no inflation adjustment) and 0.94 (following inflation adjustment). The values for the unproven bulls were 0.95 (parent average EBV and GEBV), 0.56 (blended GEBV, no inflation adjustment), and 0.88 (blended GEBV, following inflation adjustment). The use of trait-specific weighted EDM provided a 1 to $2 \%$ improvement in the accuracy of the genomic evaluations of the production traits and no improvement in the nonproduction traits. The hybrid method of estimating GEBV was found to be considerably less biased and generally more accurate than the previously used blended genomic breeding value.

Key words: genomic evaluation, deregression, single nucleotide polymorphism selection

\section{INTRODUCTION}

Genomically enhanced breeding values (GEBV) for dairy cattle have been published in New Zealand (NZ) since 2008. Until August 2013, the calculation and publication of the evaluations was overseen by New Zealand Animal Evaluation Ltd., an industry-funded organization that is tasked with ensuring optimum genetic improvement of the NZ dairy herd. Evaluations were calculated using a multistep method in which the traditional EBV were blended with direct genomic values (DGV) using a selection index approach, as originally described by VanRaden (2008). The DGV were obtained using BLUP with a genomic relationship matrix (GRM), and the phenotype was the deregressed breeding value (DRBV). Genomic information on females was not included in the analyses, other than that of a limited number of bull dams. The resulting GEBV were inflated, possibly a result of not distinguishing between alleles that are identical by descent and identical in state. The blended method did not have a built-in system of controlling inflation and adjustments were made to the GEBV postcalculation.

Because New Zealand Animal Evaluation Ltd. ceased to calculate and publish genomic evaluations, the decision was made at LIC (an AI company) to develop its own in-house genomic evaluation. A project was 
undertaken to assess methodologies to calculate GEBV that would overcome some of the deficiencies of the previous system. These include the need for the (possibly ad hoc) blending of the traditional and direct genomic EBV, the postcalculation inflation adjustments, and not utilizing the increasing number of female genotypes that are becoming available. The desired outcome of overcoming the deficiencies is reduced bias and increased accuracy of the GEBV over the previous system. An alternative to the multistep procedure is the single-step (SS) method, first proposed by Misztal et al. (2009). The method entails augmenting the pedigree-based relationship matrix by a GRM that is then incorporated into the mixed model equations. Aguilar et al. (2010) enhanced the SS method by modifying the augmented relationship matrix to adjust for the scale of the genomic predictions, thereby providing a way to control inflation of the GEBV. Legarra and Ducrocq (2012) outlined a method of implementing the SS approach within the traditional national evaluation system. This method was tested using genomic information on 5,402 bulls within the NZ national evaluation (Harris et al. 2012). However, attempts to include the genomic information on more than 50,000 cows resulted in convergence problems when solving the mixed model equations and the method was deemed infeasible for routine evaluation at present.

Another approach that uses the augmented pedigree matrix, but is much more computationally feasible than the SS method, was proposed by Harris et al. (2011). The method includes all genotyped bulls and cows as well as all their ancestors. Hence, the size of the equations is considerably smaller than that of the full national SS method. The dependent variable is the DRBV from the traditional EBV analyses. In addition to being computationally feasible to solve, this methodology retains the advantages of providing a means of controlling bias within the evaluation and obviates the need for blending of the DGV with the traditional EBV. This method will be referred to as the hybrid analysis because it uses the augmented pedigree matrix and the EBV from the traditional method. The current paper describes LIC's hybrid genomic evaluation system and compares the resulting GEBV to those obtained from the previous system of blending the DGV and traditional EBV. Additionally, results from applying the hybrid method to the national data and tracking of the youngest cohort of bulls are discussed.

\section{MATERIALS AND METHODS}

Our study was divided into 3 parts. The first was the validation of the hybrid method. This entailed treating young proven bulls as test animals, predicting their EBV using the pedigree and genomic information, and comparing the predictions to the realized EBV obtained using pedigree and progeny information. The second part was a description of the hybrid system applied to the national data using all available phenotypic information, with comparisons between the traditional EBV and blended and hybrid GEBV for proven and unproven sires. The third part tracked the youngest cohort of bulls as they received their first proof to assess the advantage of using genomic information in addition to parent average to select unproven bulls.

\section{Genotypes and Phenotypes}

For animals born in 2011 and earlier, the genotypes were from the Illumina BovineSNP50 BeadChip (Illumina Inc., San Diego, CA). A total of 34,963 autosomal SNP were retained after removing SNP for low call rates, minor allele frequencies $<2 \%$, non-Mendelian inheritance, failed Hardy-Weinberg tests, and low imputation accuracy. Editing procedures were based on the work of Wiggans et al. (2009). In 2012, a total of 1,903 bulls were originally genotyped on the GGP Illumina LD BeadChip (Illumina Inc.). Based on their genomically enhanced genetic evaluation before receiving daughter information, 228 males were selected as candidates to enter progeny testing. These males were subsequently genotyped on the Illumina BovineSNP50 BeadChip. The genotypes of the remaining 2012-born males were imputed to the selected SNP on the $50 \mathrm{~K}$ panel using Beagle 3.3.2 (Browning and Browning, 2007).

The 7 traits in the national breeding objective [breeding worth (BRW)] were considered in the validation part of this study; they are milk volume (L), fat yield $(\mathrm{kg})$, protein yield $(\mathrm{kg})$, SCS $\left[\log _{2}(\mathrm{SCC})\right]$, live weight (kg), fertility (\%), and total longevity (d), (hereafter referred to as milk, fat, protein, SCS and live weight, fertility, and longevity). The phenotype for all analyses was the DRBV for each trait as described herein.

The BRW is calculated by weighting the EBV for each of the 7 traits by its relative economic weight. The traits are as listed previously, except that the BRW includes residual survival, not longevity. Longevity is survival to the fifth lactation and residual survival is the total longevity EBV corrected for milk volume, fat yield, protein yield, SCS, live weight, and fertility. The relative economic weights that are used to weight the traits in the BRW can be obtained from the DairyNZ website (http://www.dairynz.co.nz/animal/animalevaluation/). The BRW calculated using the GEBV, instead of the traditional EBV, will be referred to as genomically estimated BRW (GEBW). 
Table 1. Frequencies of training and test sires (no.) by breed

\begin{tabular}{lcc}
\hline Breed & Test & Training \\
\hline Friesian & 685 & 3,611 \\
Jersey & 391 & 1,816 \\
Friesian-Jersey cross & 334 & 367 \\
\hline
\end{tabular}

\section{Animals for Model Validation}

The data for model validation consisted of all of LIC's genotyped bulls born in 2007 or earlier, a selection of CRV Ambreed (another AI company in Hamilton, New Zealand) genotyped bulls and genotyped females born in 2006 or earlier. All bulls were progeny tested. The genomic analyses were run using the phenotypic data that would have been available at the end of season 2008. In NZ, a season starts in June and ends in May of the next year. Calving is concentrated in July and August; inseminations take place in October and November; cows are dried off by May. Hence, an animal calving in season 2008 finishes her lactation in 2009 . Sires born in seasons 2005, 2006, and 2007 whose firstcrop daughters completed their first lactations in seasons 2009, 2010, and 2011, respectively, were the test population. Their genotypes, but not their phenotypes, were included in the analyses. Sires born in 2008 were not used as part of the test population even though their daughters completed their first lactation in season 2012. The reason was that these daughters would not contribute to the longevity EBV, as the trait is based on information available after the first lactation. Genotyped sires born before 2005 will be referred to as the training sires. The accuracy of prediction was calculated as the correlation between the traditional EBV (obtained using data available at the end of season 2013) and GEBV of the test sires. The inflation was assessed using the slope of the regression of traditional EBV on GEBV, a slope of unity indicating no inflation.

Table 1 shows the breed composition of the training and test sire populations. Table 2 shows the year of birth by breed for the test sires. We distinguish between 3 breeds, 2 purebred (Friesians and Jerseys) and 1 crossbred breed [Friesian-Jersey (FJ) crosses]. The purebreds are at least 13/16 of the specified breed. The training data set contained genotypes on almost 20,000 cows, with the majority (89\%) born in 2005 and 2006 . These cows were from specialized progeny test herds. Prior to 2005, the female genotypes were available on bull dams.

\section{Animals for National Evaluation}

Following model validation, GEBV were calculated for the national population for the 7 traits in the na- tional breeding objective. The DRBV were derived from traditional EBV that were available in June 2013. The national evaluation consisted of all genotyped animals born in 2012 or earlier and their ancestors. This was an increase of approximately 6,000 genotyped males and 27,000 genotyped females compared with the validation data. Daughters of bulls born in 2008 completed their first lactation by May 2013. Bulls born in 2009 and later had no daughter records at the time of this analysis.

\section{Tracking the Young Bulls}

The hybrid evaluation was implemented in September 2013. Daughters of bulls born in 2009 started their lactations in July and August of 2013 and completed their first lactation by May 2014. These bulls were evaluated in June 2013, before their daughters' calving, using the traditional evaluation and the genomically enhanced evaluation before their daughters' first lactation and then throughout the season (September, November, February, and April). This provided an opportunity to track the reliabilities of their proofs as their daughters received lactation information and to determine how the 2 initial proofs compared with the end-of-season proofs.

\section{Blended Breeding Value}

This section summarizes the method of Harris and Johnson (2010). The DGV were estimated using an across-breed BLUP that included the GRM that was adjusted for breed frequencies as outlined by Harris and Johnson (2010). Genotypes were included for all bulls and a limited number of bull dams. The phenotype was the DRBV, calculated as shown below.

The EBV obtained by solving the mixed model equations that use only pedigree and progeny information is

$$
\widehat{\mathbf{B V}}=\hat{\mathbf{v}}+\mathbf{Q} \hat{\mathbf{b}}
$$

where $\hat{\mathbf{v}}$ is the vector of animal solutions, $\hat{\mathbf{b}}$ is a vector of breed genetic group solutions, and $\mathbf{Q}$ is matrix that relates base animals to their respective population means, as originally defined by Quaas (1988). Then $\hat{\mathbf{v}}$ is the vector of national EBV for genotyped animals and

Table 2. Frequencies of test sires (no.) by breed and year of birth

\begin{tabular}{lrcc}
\hline Breed & 2005 & 2006 & 2007 \\
\hline Friesian & 251 & 238 & 196 \\
Jersey & 140 & 139 & 112 \\
Friesian-Jersey cross & 76 & 107 & 151 \\
\hline
\end{tabular}


their ancestors, after subtracting the fixed effect of the genetic group; $\mathbf{A}$ is the additive genetic relationship matrix based on pedigree [hereafter referred to the numerator relationship matrix (NRM)] for this set of animals. The deregressed proofs, $\mathbf{y}$, for these animals can be obtained from

$$
\mathbf{D} \hat{\mathbf{y}}=\left(\mathbf{D}+\mathbf{A}^{-1}\right) \hat{\mathbf{v}}
$$

where $\mathbf{D}=\operatorname{diag}[\mathbf{r} /(1-\mathbf{r})]$ and $\mathbf{r}$ is the vector of deregressed reliabilities (Appendix A in Harris and Johnson, 2010). For example, for a bull with no genotyped daughters, the corresponding element of $\mathbf{D}$ is proportional to the effective number of daughters and the element of $\mathbf{y}$ is 2 times the daughter yield deviation. For a bull with all daughters genotyped or a young bull with no daughters, the corresponding elements of $\mathbf{D}$ and $\mathbf{D} \hat{\mathbf{y}}$ are zero. For a cow with no daughters, the deregressed information represents the cow's own production.

Only phenotypes from bulls were included in this evaluation. Phenotypes from dams were set to zero to prevent selection bias from affecting the DGV. The GEBV were obtained by blending the DGV and the traditional EBV using a selection index method as outlined by VanRaden (2008).

The blended GEBV was adjusted a posteriori for inflation by scaling the Mendelian sampling (MS) component of the GEBV. This was calculated as the difference between the GEBV and the parent average part of the GEBV. The adjustment was derived by regressing the MS estimate obtained from the daughter-proven EBV on the MS estimate obtained from the GEBV. The estimated regression coefficient was used to adjust the MS estimate of the GEBV before adding it back to the parent average of the GEBV for each trait.

The reliabilities of the blended EBV were calculated as follows:

$$
\mathbf{R}_{\mathrm{G}}=\frac{\left[\mathbf{G}\left(\mathbf{G}+\mathbf{D}_{2}^{-1}\right)^{-1} \mathbf{G}\right]_{i i}}{\mathbf{G}_{i i}},
$$

where $\mathbf{G}$ is the genomic relationship matrix, and $\mathbf{D}_{2}$ is the partition of $\mathbf{D}$ pertaining to genotyped animals. Based on selection index, $\mathbf{R}_{\mathrm{G}}$ is combined with the corresponding reliability $\mathbf{R}_{\mathrm{A}}$ using the $\mathbf{A}_{22}$ matrix in place of $\mathbf{G}$ above and the reliability from the national evaluations $\mathbf{R}_{\mathrm{N}}$ to give a total genomic reliability, $\mathbf{R}_{g}$

$$
\mathbf{R}_{g}=\frac{\mathbf{R}_{\mathrm{G}}-\mathbf{R}_{\mathrm{A}}+\mathbf{R}_{\mathrm{N}}-2 \mathbf{R}_{\mathrm{G}} \mathbf{R}_{\mathrm{N}}+\mathbf{R}_{\mathrm{G}} \mathbf{R}_{\mathrm{A}} \mathbf{R}_{\mathrm{N}}}{1-2 \mathbf{R}_{\mathrm{A}}-\mathbf{R}_{\mathrm{N}} \mathbf{R}_{\mathrm{G}}+\mathbf{R}_{\mathrm{A}} \mathbf{R}_{\mathrm{G}}+\mathbf{R}_{\mathrm{N}} \mathbf{R}_{\mathrm{A}}}
$$

[equations 29 and 32 in Harris and Johnson (2010)].

\section{Hybrid Method}

The NRM augmented by the genomic relationship matrix $(\mathbf{G})$ is referred to as the $\mathbf{H}$ matrix. The $\mathbf{H}$ matrix inverse is described by Legarra et al. (2009), and a computationally feasible way of calculating its inverse is outlined by Aguilar et al. (2010). A genomic evaluation system that incorporates the inverse of $\mathbf{H}$ and uses traditional national EBV, based on pedigree, as the starting point, was developed. The method uses the DRBV and reverses the deregression, replacing the inverse of the NRM by the inverse of the $\mathrm{H}$ matrix as follows:

$$
\hat{\mathbf{g}}=\left[\left(\mathbf{D}+\mathbf{H}^{-1}\right)^{-1}\right] \mathbf{D} \hat{\mathbf{y}}
$$

where $\hat{\mathbf{g}}$ is the vector of animal solutions and $\mathbf{D}$ is as described for equation [2].

As summarized by Misztal et al. (2013), better accuracy and lower bias of GEBV can be obtained by finetuning some of the parameters in $\mathbf{H}$. Two parameters were used: a scaling parameter, $\varphi$, (equation A3 in the Appendix) and a mixing parameter, $\theta$, (equation A2 in the Appendix), which is analogous to $\lambda$ in Aguilar et al. (2010). Three levels $(0.3,0.5,0.7)$ of the scaling parameter were investigated. The mixing parameter was 0.80 for all evaluations. Our approach is similar to the one-step blending of Su et al. (2012), who used a mixing parameter of 0.80 , but did not include a scaling parameter. Misztal et al. (2013) indicated that, in general, the best accuracies and biases are obtained by using a mixing parameter of around 0.80 .

This procedure will be referred to as the hybrid method in the sense that the pedigree-based relationship matrix is augmented by the genomic relationship matrix and a single analysis is performed to obtain GEBV. This differs from the selection index method, where separate analyses are done to calculate the traditional EBV and DGV , incorporating the pedigree-based relationship

Table 3. Number of regions and number of SNP selected for each trait

\begin{tabular}{lcc}
\hline Trait & $\begin{array}{c}\text { No. of } \\
\text { regions }\end{array}$ & $\begin{array}{c}\text { No. of } \\
\text { SNP }\end{array}$ \\
\hline Milk & 18 & 355 \\
Fat & 16 & 441 \\
Protein & 15 & 386 \\
SCS & 14 & 434 \\
Live weight & 9 & 382 \\
Fertility & 13 & 416 \\
Longevity & 11 & 456 \\
\hline
\end{tabular}


Table 4. Proportion of variance associated with the numerator relationship matrix (NRM), the Euclidean distance (EDM) for SNP not in the selected regions and for SNP in the top 5 selected regions

\begin{tabular}{lcccc}
\hline & & \multicolumn{2}{c}{ Regions } \\
\cline { 3 - 4 } Trait & NRM & EDM & No. of SNP & EDM \\
\hline Milk & 0.028 & 0.65 & 115 & 0.14 \\
Fat & 0.054 & 0.66 & 146 & 0.13 \\
Protein & 0.045 & 0.69 & 170 & 0.11 \\
SCS & 0.034 & 0.74 & 195 & 0.09 \\
Live weight & 0.058 & 0.71 & 283 & 0.16 \\
Fertility & 0.067 & 0.68 & 155 & 0.11 \\
Longevity & 0.249 & 0.57 & 218 & 0.09 \\
\hline
\end{tabular}

matrix or genomic relationship matrix, respectively, which are then combined to obtain the GEBV.

The conjugate-gradient method with iteration on data (Strandén and Lidauer, 1999) is used to solve these equations after first using matrix inversion techniques to calculate the inverses of $\mathbf{G}$ and $\mathbf{A}_{22}$ (the NRM of the genotyped animals).
The advantages of using equation [3] are that the set of equations that need to be solved is considerably smaller than those of a full SS model and the same model can be used for all traits. For the national analyses, there were 60,842 genotyped animals $(13,268$ males and 47,574 females) and a total of 240,321 animals (27,223 males and 213,098 females) when includ-

Table 5. Selected top 5 genomic regions for production traits and nonproduction traits, with map positions and references to researchers who identified associations for these regions

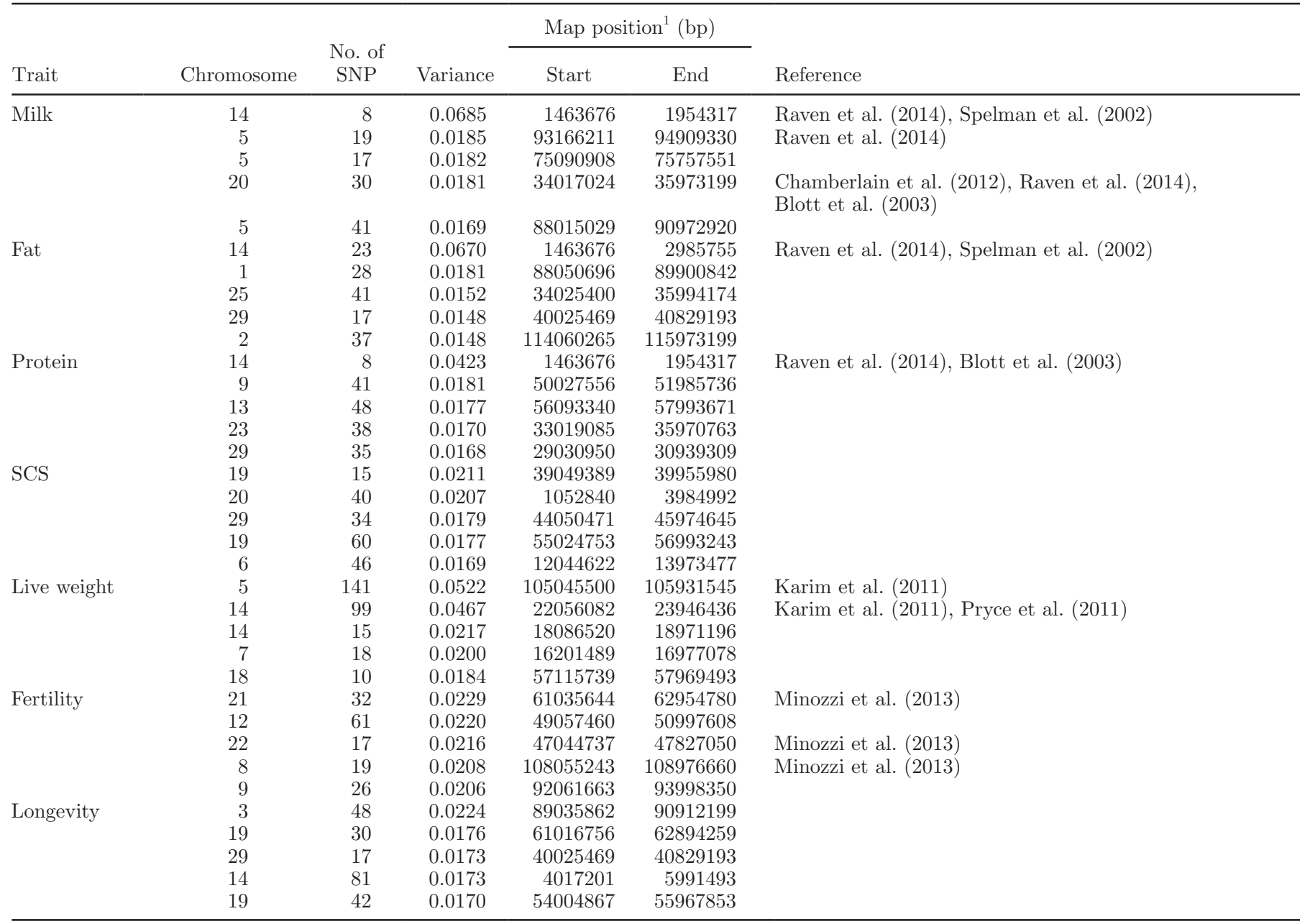

${ }^{1}$ These positions were obtained from dbSNP BUILD 140 (http://www.ncbi.nlm.nih.gov/projects/SNP/). 
ing ancestors. A full national analysis in NZ (i.e., to calculate traditional EBV) includes approximately 25 million animals.

Calculation of reliability uses matrix inversion. The coefficient matrix can be written in partitioned form as

$$
\left[\begin{array}{cc}
\mathbf{D}_{1}+\mathbf{A}^{11} & \mathbf{A}^{12} \\
\mathbf{A}^{21} & \mathbf{D}_{2}+\mathbf{H}^{22}
\end{array}\right]
$$

and the genotyped partition of the inverse of this matrix is

$$
\mathbf{C}^{22}=\left[\mathbf{D}_{2}+\mathbf{H}^{22}-\mathbf{A}^{21}\left(\mathbf{D}_{1}+\mathbf{A}^{11}\right)^{-1} \mathbf{A}^{12}\right]^{-1} .
$$

The reliabilities of genotyped individuals are then given by the diagonal elements of $\mathbf{I}-\mathbf{C}^{22}$. Matrix manipulation is difficult in the case of ancestors. Instead, the reliabilities of ungenotyped ancestors can be updated for the genomic information provided by their progeny using methods outlined in Harris and Johnson (1998). Three steps are involved; first, the reliability of genotyped animals that arises from genomics is extracted using equation 2 of Harris and Johnson (1998). Then, genomic reliability from progeny is then passed up to their ancestors using their equation 5. Finally, the genomic reliability is combined with the original reliability using the equation that is shown in step 3 of Harris and Johnson (1998).

The GEBV for nongenotyped descendants are then obtained using the technique outlined in Harris and Johnson (2010). The hybrid GEBV is obtained as shown in equation [1] but with the $\hat{\mathbf{g}}$ from equation [3] replacing $\hat{\mathbf{v}}$. This hybrid method avoids the blending step used in the previous method of genomic evaluation.

\section{Genomic Relationships}

The Appendix outlines 2 ways of calculating genomic relationships resulting in either the Euclidean distance matrix (EDM) or the GRM using allele cross-products. The evaluations were initially attempted by forming an $\mathbf{H}$ matrix in which the pedigree-based relationship matrix was augmented with the GRM. The construction of the GRM requires knowledge of the allele frequencies in the base populations of each breed. These can be obtained using the approach of Harris and Johnson (2010), but the method is computationally intractable for populations of greater than 10,000 animals. Instead, the GRM was formed using gene frequencies that were estimated using the method of Makgahlela et al. (2013). Convergence monitoring when solving equation [3] had occasionally identified some instability, perhaps due to near singularity conditions for the GRM. Subsequently,

Table 6. Inflation (in units of the given trait) and accuracy of the blended and hybrid breeding value (BV) estimated using 3 different scaling parameters $(\varphi)$

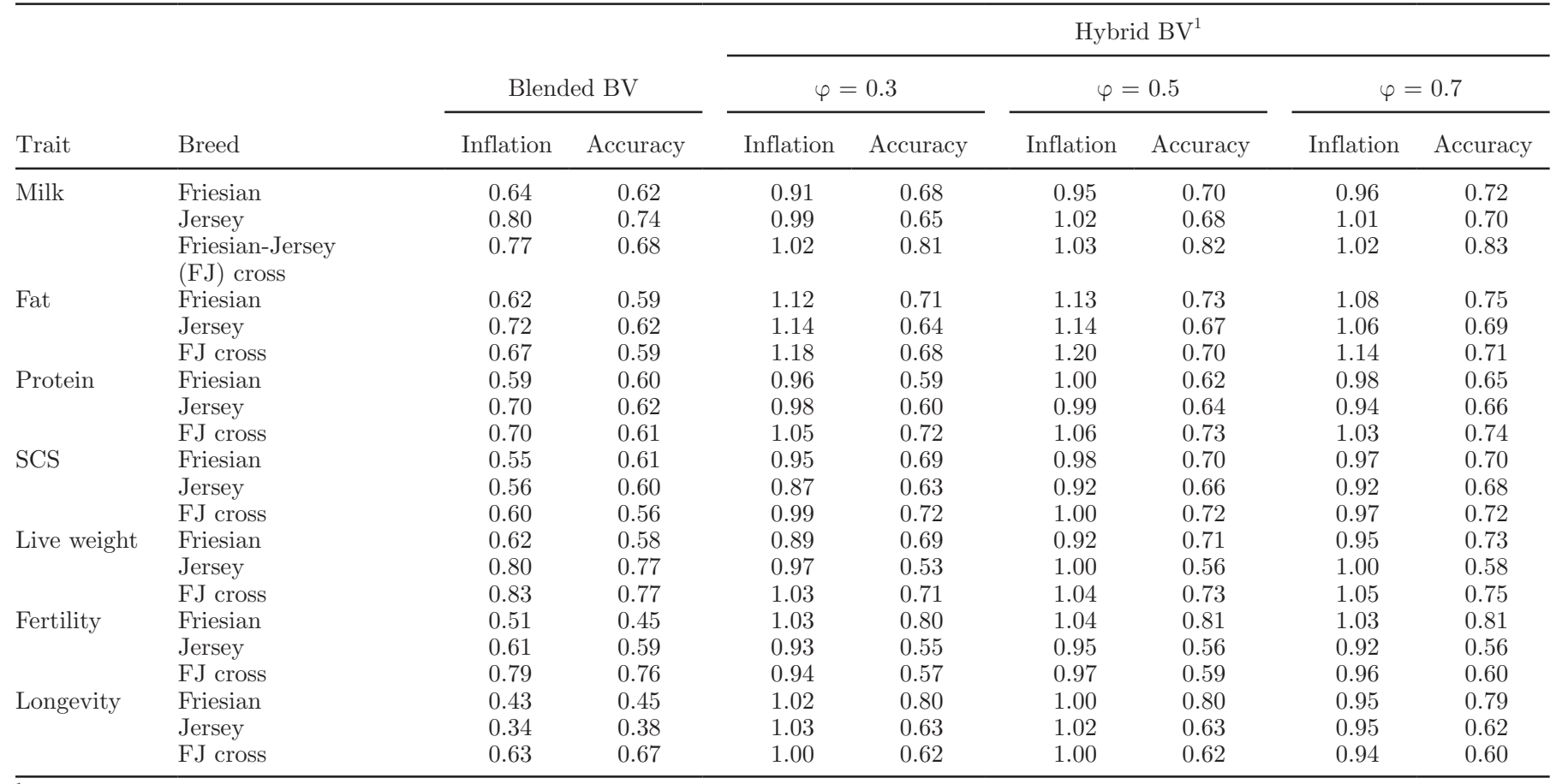

${ }^{1} \mathrm{~A}$ mixing parameter, $\theta$, of 0.80 was used for the hybrid BV. 
evaluations in which the GRM was replaced with the EDM were attempted. The EDM has the advantage of having nonnegative relationships and of being positive definite provided no 2 genomes are identical. The EDM, which involves differences, is less affected by breed and no breed adjustment was attempted. A comparison of the GEBV resulting from the 2 relationship matrices showed the evaluations using the EDM had accuracies that were 1 to $2 \%$ higher and the inflation estimates that were 1 to $5 \%$ lower than those obtained with the GRM. Therefore, the definition of genomic relationship matrix based on EDM is used in this study.

\section{SNP Selection}

Genome-wide association studies (GWAS) were done to determine if specific regions of the genome had higher association to the traits in BRW than other regions of the genome. The male and female populations were analyzed separately. The phenotype (dependent variable) for the males was the DRBV. The dependent variable for the females was the phenotypic record adjusted for fixed effects and random effects. In all cases, the phenotype was adjusted for the fixed effects of breed and breed heterozygosity and the random effect of contemporary group. Additional adjustments were trait dependent. For milk, fat , and protein, the phenotypic record was the yield deviation (Johnson, 1996) adjusted for the fixed effects of month after the start of the planned start of calving within contemporary group, induction of calving, season (spring or autumn), and age at calving, in months, within breed group. For SCS, the averaged herd test record was adjusted for the same effects as yield deviations. Fertility was adjusted for stage of lactation (in days) and age at calving (in months). The live weight phenotype was adjusted for stage of lactation (in days) and its quadratic term as well as age-by-breed effects with their quadratic terms.

Bayes C $\pi$ (Habier et al., 2011) analyses were run for each trait to obtain the genotypic and residual variances. These variances were used in the subsequent analyses to estimate the magnitude of the effects associated with specific regions of the genome using Bayes B (Meuwissen at al., 2001) and implemented through GenSel (Fernando and Garrick, 2009).

For each trait, the top 100 regions (based on the magnitude of the effect) from the analyses of using the male and female populations were selected for comparison. Regions that matched exactly, overlapped, or were within 10 SNP of each other were chosen to be included as separate effects in a reducing kernel Hilbert space (RKHS; Gianola and van Kaam, 2008) analysis. In some cases, several selected regions overlapped and they were amalgamated into one bigger region. Using the consensus from the 2 analyses (different sexes and different ways of representing the phenotypes) was done to increase the likelihood that the effects were real and not an artifact of the data or analyses. The actual number of regions and SNP chosen varied across the traits, as shown in Table 3.

The EDM were created for each of the selected regions. The variances were estimated using RKHS

Table 7. Inflation (in units of the given trait) and accuracy of the genomically enhanced breeding values (GEBV) from the unweighted and weighted hybrid analyses using the most optimal scale parameter, $\varphi$, for each trait

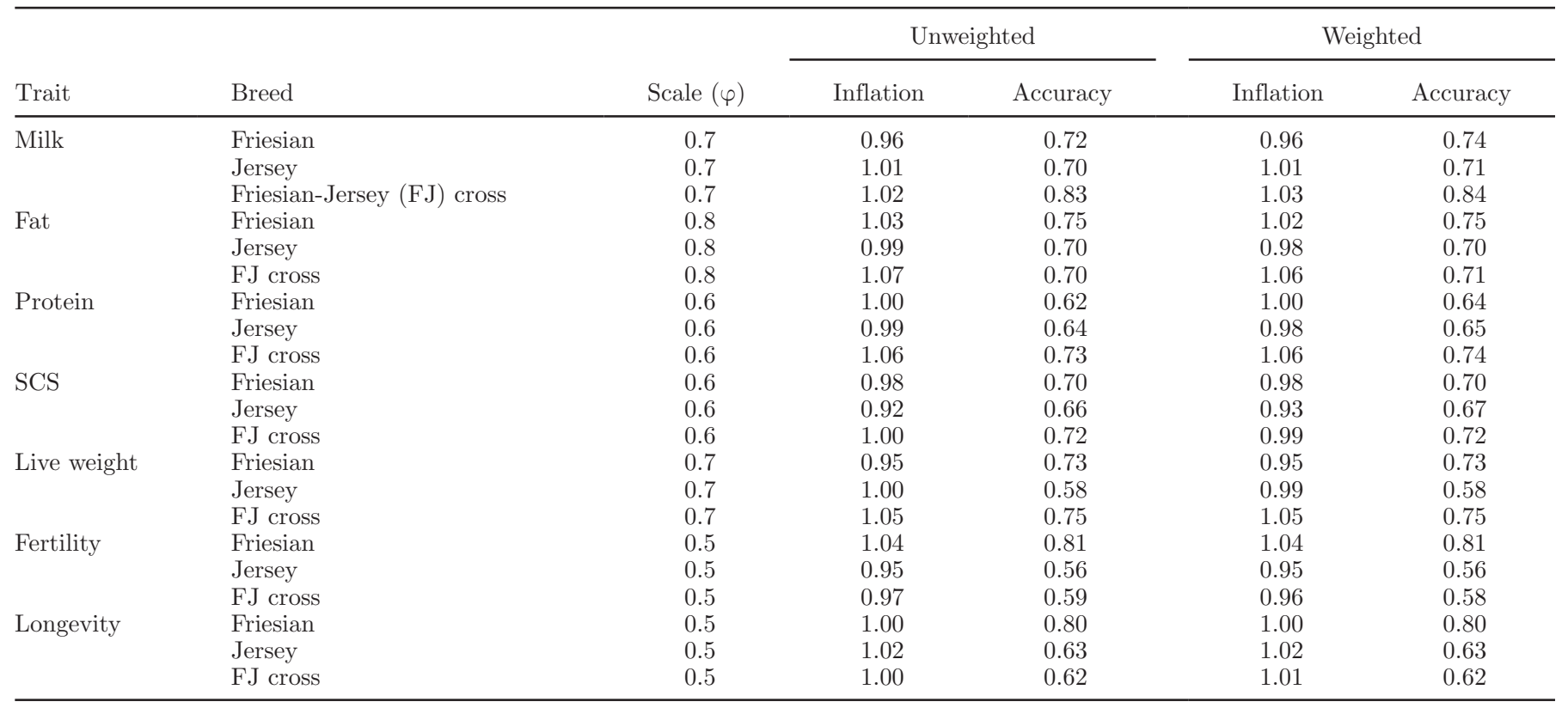



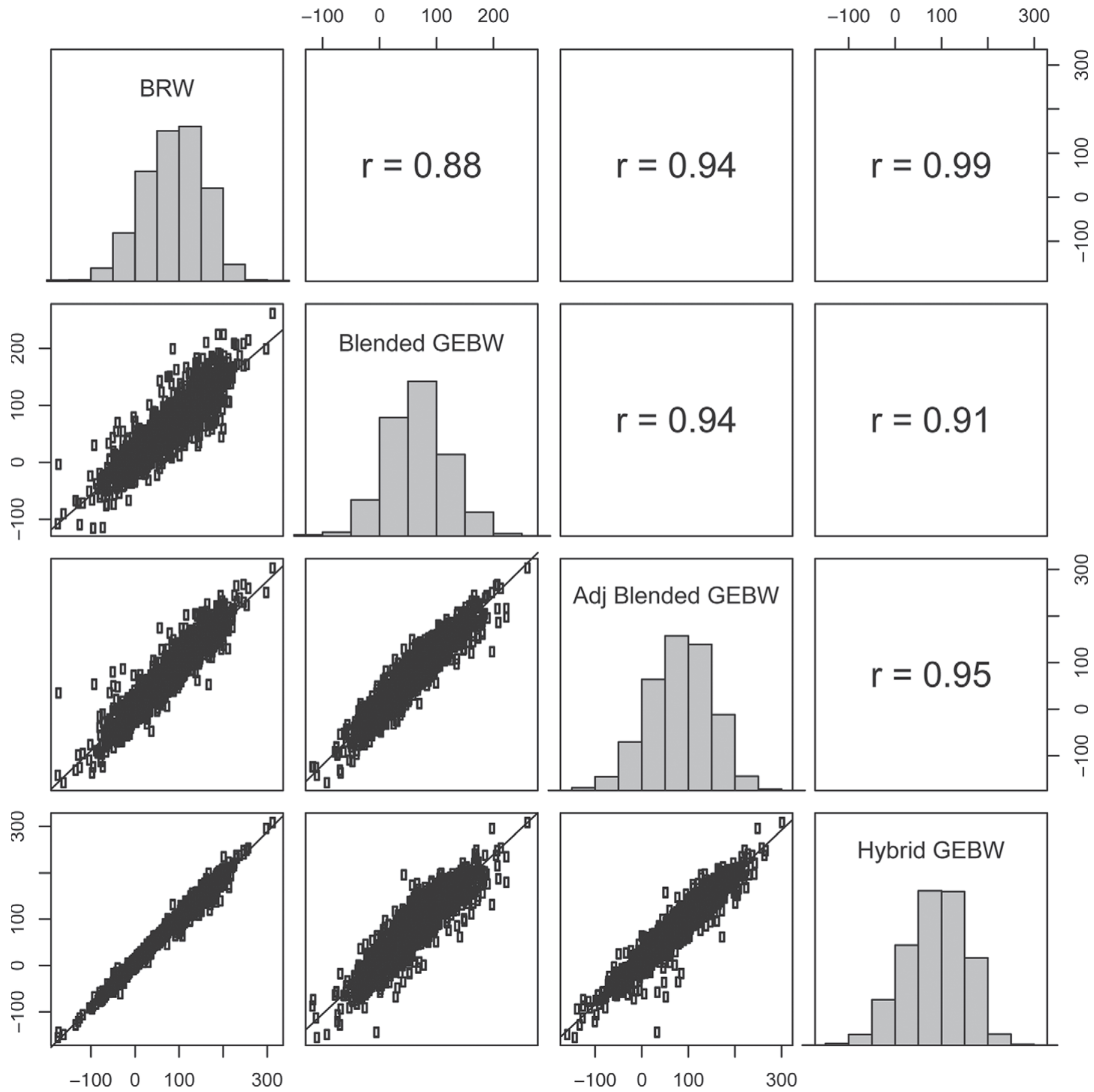

Figure 1. Frequency distributions and scatter plots for breeding worth (BRW), blended genomically estimated BRW (GEBW), inflationadjusted GEBW, and hybrid GEBW of proven Friesian sires $(\mathrm{n}=1650)$.

analyses (Gianola and van Kaam, 2008) in which each EDM, along with the NRM and the EDM created from the SNP that were not selected, were fitted as separate effects. The relative variance was estimated for each EDM. As a check of equivalence, the relative variances were used to create a weighted EDM based on all SNP, which was fitted along with the NRM, in subsequent RKHS analyses. The method for weighting the EDM, shown in the Appendix, is analogous to the method outlined by VanRaden (2008). 

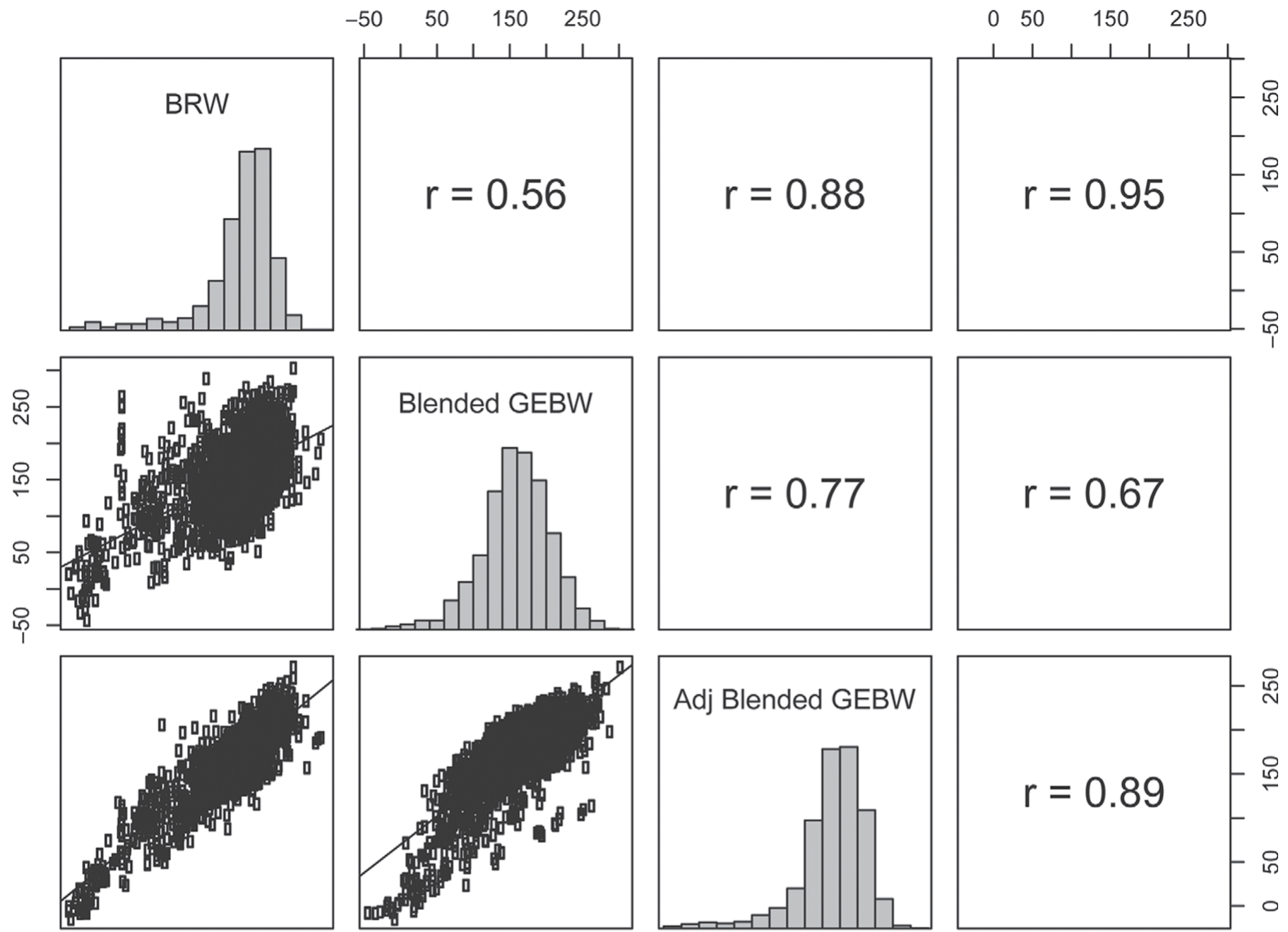

\section{1}
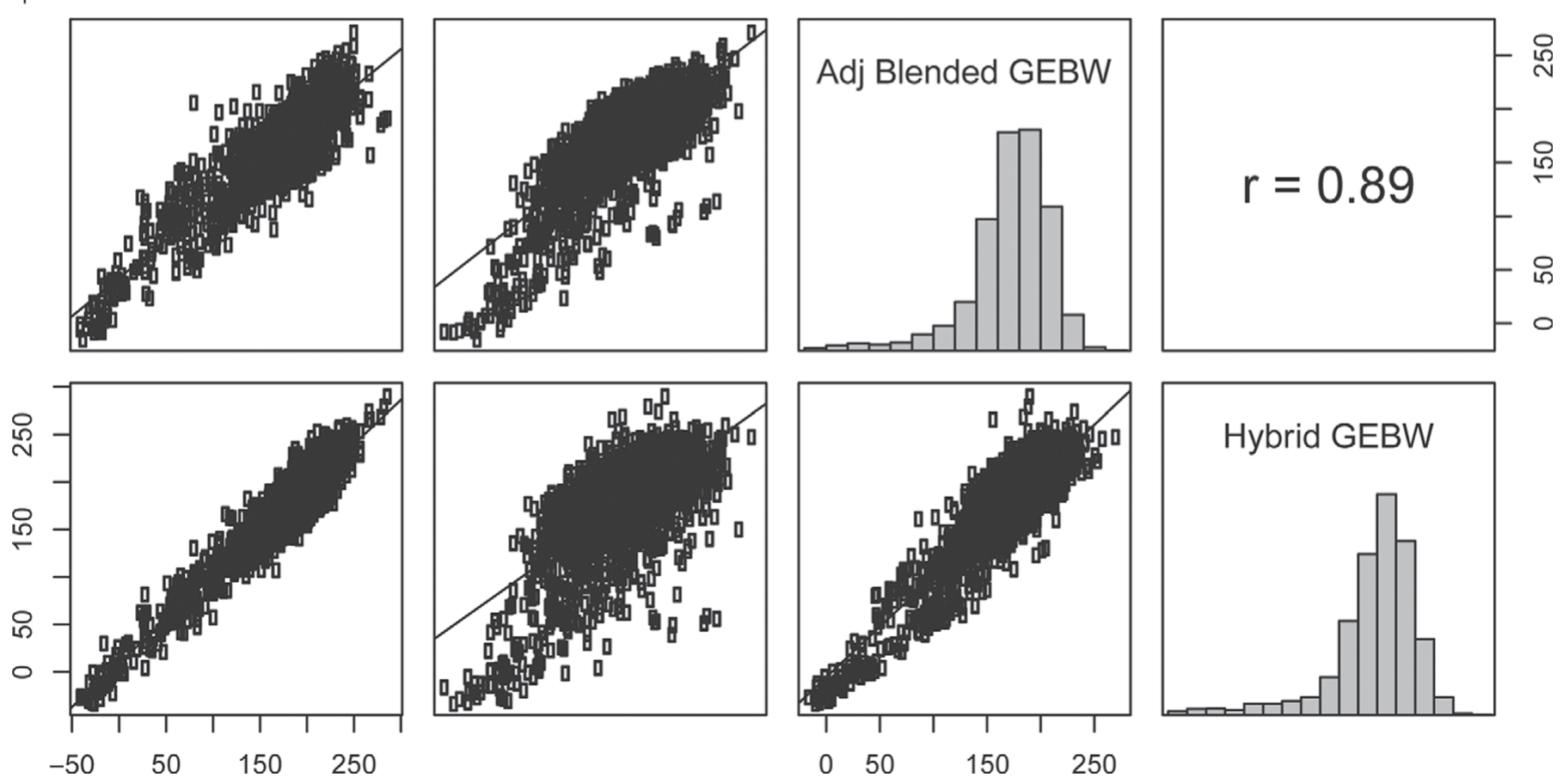

Figure 2. Frequency distributions and scatter plots for breeding worth (BRW), blended genomically estimated BRW (GEBW), inflationadjusted GEBW, and hybrid GEBW of unproven Friesian sires $(\mathrm{n}=2514)$.

\section{RESULTS}

\section{SNP Variances}

The variances associated with the NRM, EDM containing the SNP that were not in the selected regions, and the top 5 regions for each trait are shown in Table
4. The values shown are relative to the total genetic variance (i.e., variance associated with the NRM and all the EDM). The pattern was similar for all traits except longevity. The NRM accounted for 3 to $7 \%$ of the genetic variance, the EDM excluding the selected SNP accounted for 65 to $74 \%$ of the genetic variance. The 


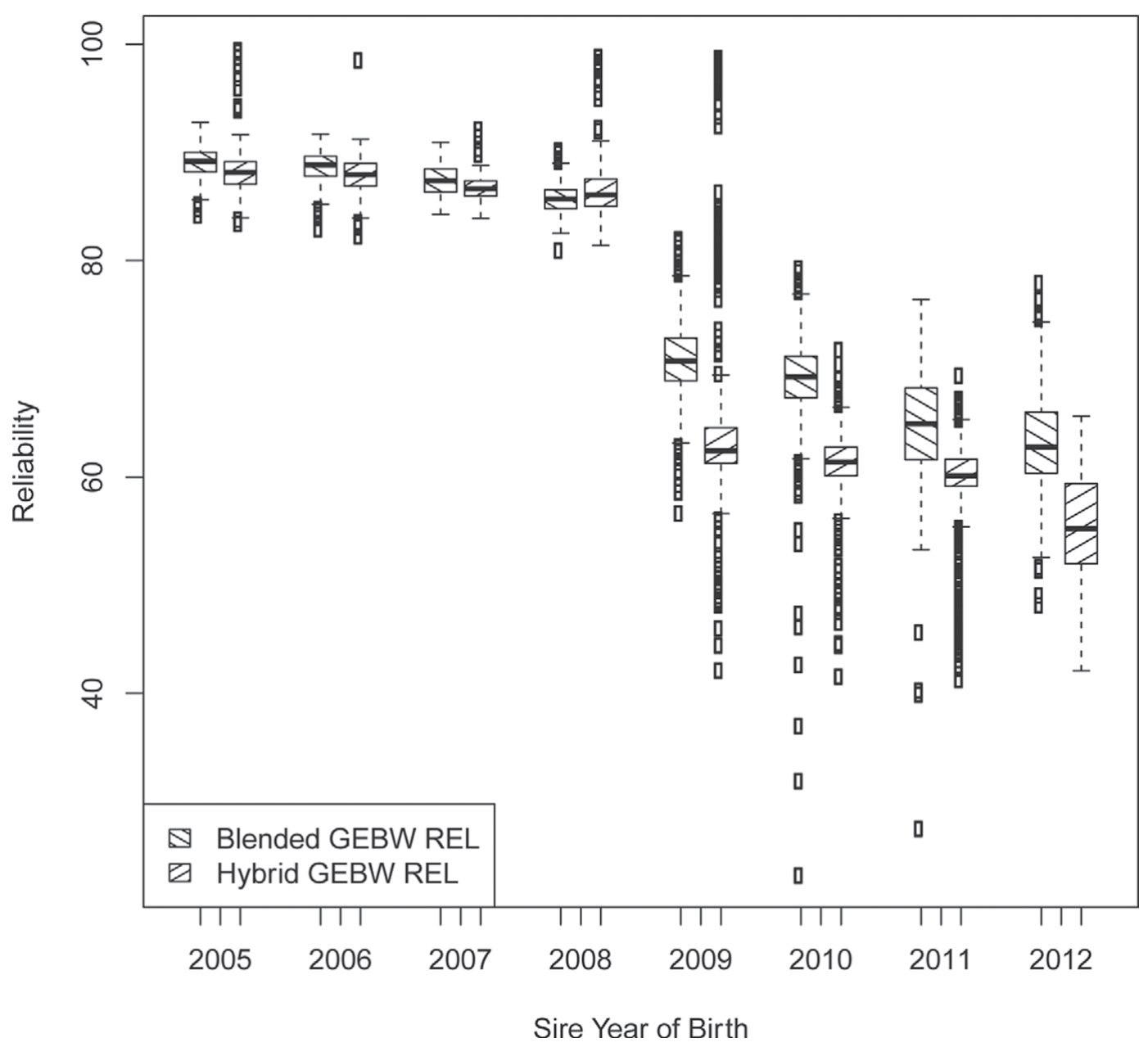

Figure 3. Reliabilities (REL) of blended genomically estimated breeding worth (GEBW) and hybrid GEBW for proven (born $\leq 2008)$ and young sires.

top 5 selected regions show that considerably less than $1 \%$ of the SNP accounted for 9 to $16 \%$ of the genetic variance. Evidence for major gene effects for all traits was observed. Longevity differed to the other traits in that the NRM accounted for $25 \%$ of the genetic variance and the EDM accounted for less variance than was the case for the other traits. Because longevity is defined as survival until the fifth lactation, animals in the analysis that have yet to express the actual record (i.e., have censored records) would have a greater proportion of their evaluation influenced by parent average. This may explain why the NRM accounts for more variation than the EDM does for this trait. Table 5 shows the map positions of the top 5 selected genomic regions for each trait and references work that has found associations similar positions to those we found. The table is indicative and not comprehensive in the sense that a thorough search of the association literature may uncover regions that match those that we have found. The regions on chromosome 14 for milk, fat, and protein contain the DGAT1 region of the chromosome. The DGAT1 mutation was found to increase milk production traits in New Zealand dairy cattle (Spelman, et al. 2002). The effect of DGAT1 on milk production traits was also found by Raven et al. (2014), as well as by other researchers. The only other region associated with increased milk production that was corroborated by others was on chromosome 20 (Chamberlain et al., 2012, Raven et al., 2014, Blott et al., 2003). Regions close to those that we identified for live weight were found on chromosome 5 by Karim et al. (2011) and chromosome 14 by Karim et al. (2011) and Pryce et al. (2011), with the region on chromosome 14 containing the PLAG1 mutation. Minozzi et al. (2013) found regions on chromosomes 8, 12, and 21 that were similar to ours for fertility. The regions identified by Meredith et al. (2013), Raven et al. (2014), and Sahana et al. (2013) to be associated with SCS were not identified in our association studies.

The correlations between the GEBV calculated with the separate EDM and the weighted EDM were $>0.99$ 


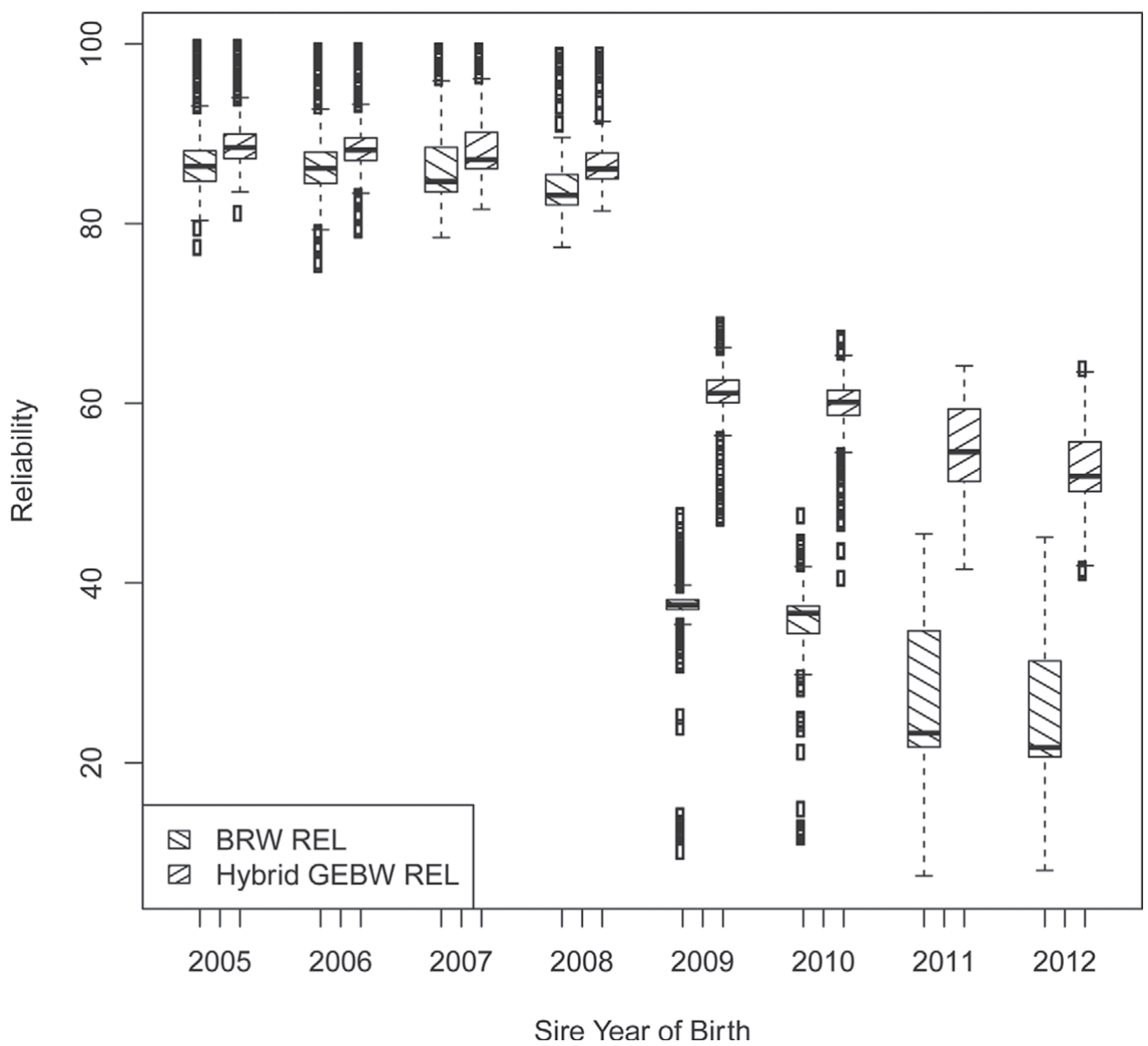

Figure 4. Reliabilities (REL) of breeding worth (BRW) and genomically estimated BRW (GEBW) for proven (born $\leq 2008)$ and young sires.

in all traits. The relative variances were used to create trait-specific weighted EDM (see Appendix). The weighted EDM were then used in the validation as part of the hybrid analyses to determine whether differential weighting of SNP increased the accuracy of the GEBV.

\section{Blended Versus Hybrid Genomic Evaluations for Test Sires}

Table 6 contains the inflation (slope) and accuracy of the GEBV of test animals evaluated using the blended and hybrid approaches. The inflation estimates for the blended GEBV are those obtained before the inflation adjustment. An inflation estimate of less than one means that the GEBV are inflated. For most of the blended BV, the estimates are substantially less than one, indicating considerable inflation. Breed-to-breed differences are greater for fertility and longevity than for the other traits. The GEBV from the hybrid analysis are considerably less inflated than the blended breeding values. The inflation estimates from the hybrid method are of similar magnitudes to those found by $\mathrm{Su}$ et al. (2012) for the 5 traits that both studies included. Our estimates of accuracy are higher than those of Su et al. (2012). The scale parameter had a small effect on the inflation and accuracy of the GEBV. Overall, a scaling parameter of 0.3 produced less desirable outcomes than the other scale parameters. The optimal scale parameter differed across the traits.

The inflation and accuracy of the test sires GEBV obtained using the weighted EDM and the chosen scale parameter for each trait are shown in Table 7 . The weighting of the EDM did little to increase the accuracy of evaluation. Milk and protein showed up to a $2 \%$ improvement and fat showed a $1 \%$ improvement only in the FJ crosses. The small improvement in accuracy may be a function of the density of the genotypes used in this study. The $50 \mathrm{~K}$ panel may not be dense enough to contain markers that are in high enough linkage disequilibrium with the causative alleles to improve 


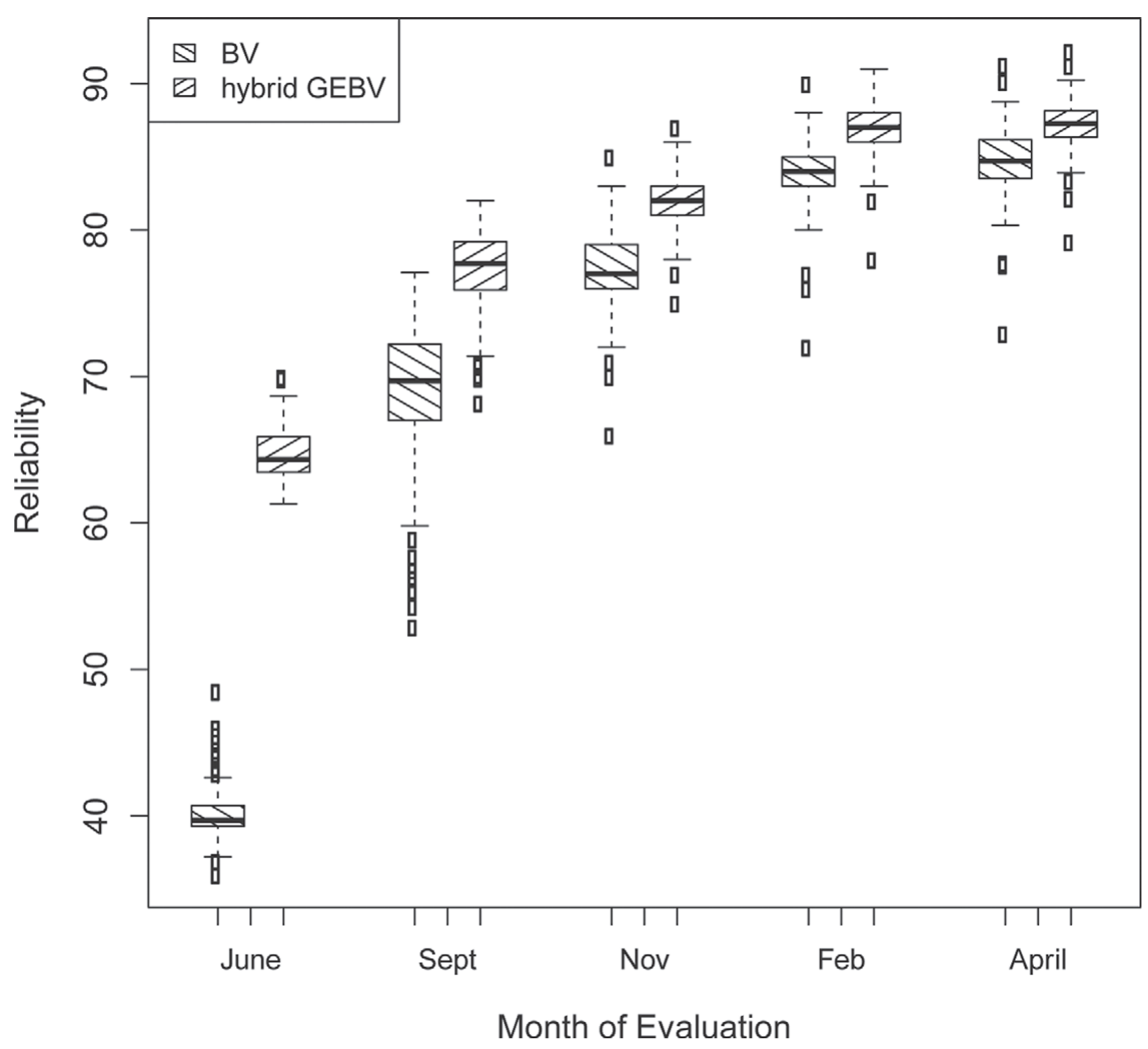

Figure 5. Reliability of milk volume breeding value (BV) and genomically enhanced breeding value (GEBV) across the season for 2009-born bulls.

prediction. The decision was made to use the weighted EDM in the national analysis for the 3 production traits and the unweighted EDM for the other 4 traits.

\section{Traditional Versus Genomic Evaluations for the National Data Set}

Frequency distributions and scatter plots of BRW, blended GEBW, inflated-adjusted blended GEBW, and hybrid GEBW of proven (born between 2000 and 2008, inclusive) and unproven (born 2009 to 2012, inclusive) Friesian sires are shown in Figures 1 and 2, respectively. The patterns for the other breeds were similar to that of the Friesians. For the proven sires, the blended GEBW had the lowest correlation (0.88) with BRW. Adjusting for inflation increased the correlation to 0.94 . The hybrid GEBW had a very high correlation (0.99) with the BRW. The correlations between the hybrid and the blended GEBW and the adjusted blended
GEBW were 0.91 and 0.95 , respectively, showing that the hybrid GEBV differ from the blended GEBV even for proven sires. Given that genotypes would have little information to add to the proven sire evaluations, a close relationship between BRW and GEBW is expected. Blended GEBV account for genetic relationships only through the GRM and therefore may not account for all the genetic associations among animals. Hence, they are less regressed than the hybrid GEBV that are calculated using both additive genetic and genomic relationships, with the contribution from the latter scaled to control inflation.

For unproven sires, the genomic information adds MS deviation information to the parent average information so the association between the BRW and GEBW is expected to be lower than is the case for proven sires. The correlation between the BRW and blended GEBW was 0.56 . The correlation increased to 0.88 after adjusting for inflation. As was found for the proven bulls, the 


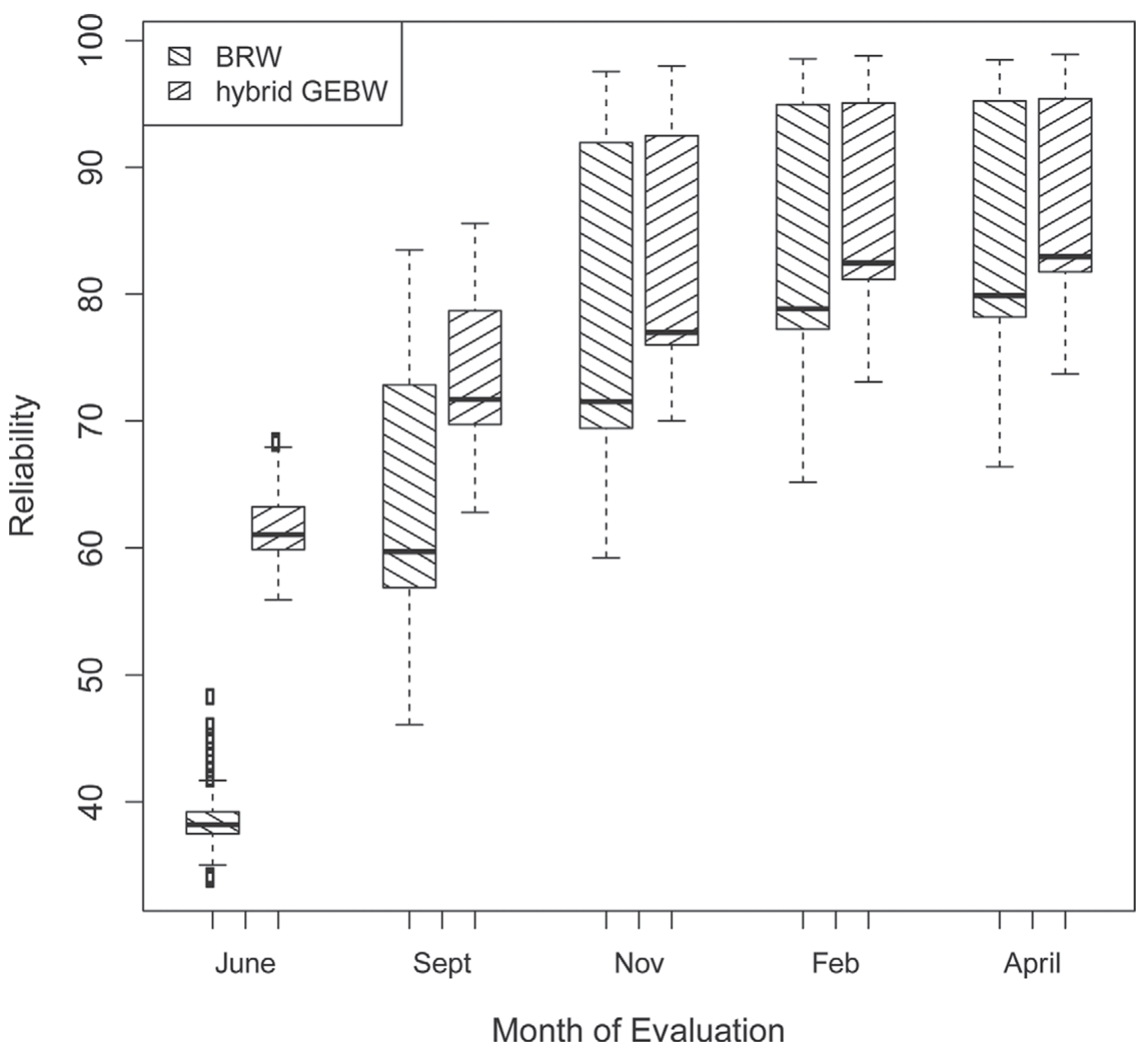

Figure 6. Reliability of breeding worth (BRW) and genomically estimated BRW (GEBW) across the season for 2009-born bulls.

BRW and hybrid GEBW were more closely correlated (0.95) to each other than they were to the blended GEBW $(\leq 0.89)$. Even though the correlation between the BRW and GEBW is high, the genomic data adds information that creates differentiation among unproven bulls, thereby improving the predictive ability.

Figure 3 contains the reliabilities calculated for the blended and hybrid GEBW for sires born between 2005 and 2012. Sires born in 2008 and earlier are proven. The differences are small (less than 1\%) for the proven sires. The average reliabilities of the blended GEBW were 5 to $8 \%$ higher than those of the hybrid GEBW for the unproven bulls. This is not an indication of the superiority of the blended method, as can be seen in Table 6, but rather an indication that the blended method produced inflated estimates of reliability.

The reliabilities of the BRW and hybrid GEBW of bulls born from 2005 to 2012 are shown in Figure 4. For the proven bulls, the average reliabilities of the GEBW were slightly higher $(<2.5 \%)$ than those of the
BRW. For unproven bulls, the reliability of the GEBW was considerably higher than that of the BRW. The mean difference between the reliabilities of the GEBW and BRW of bulls born in 2009 and 2010 was $24 \%$, on average. The mean difference was $28 \%$ for bulls born in 2011 and 2012. A large percentage of the bulls born in the latter $2 \mathrm{yr}$ are themselves the progeny of unproven sires, with $57 \%$ of bulls born in 2011 and $72 \%$ of bulls born in 2012 from unproven sires. The low GEBW reliabilities of these sires results in a lower reliability of their progeny.

\section{Tracking of Young Sires}

The reliabilities of traditional and genomically enhanced evaluations of the 2009-born sires were tracked over the season in which their first-crop daughters calved. Figure 5 contains the boxplots for the reliability of the EBV and hybrid GEBV for milk volume from June 2013 until April 2014. Figure 6 contains the re- 


\section{Friesians}

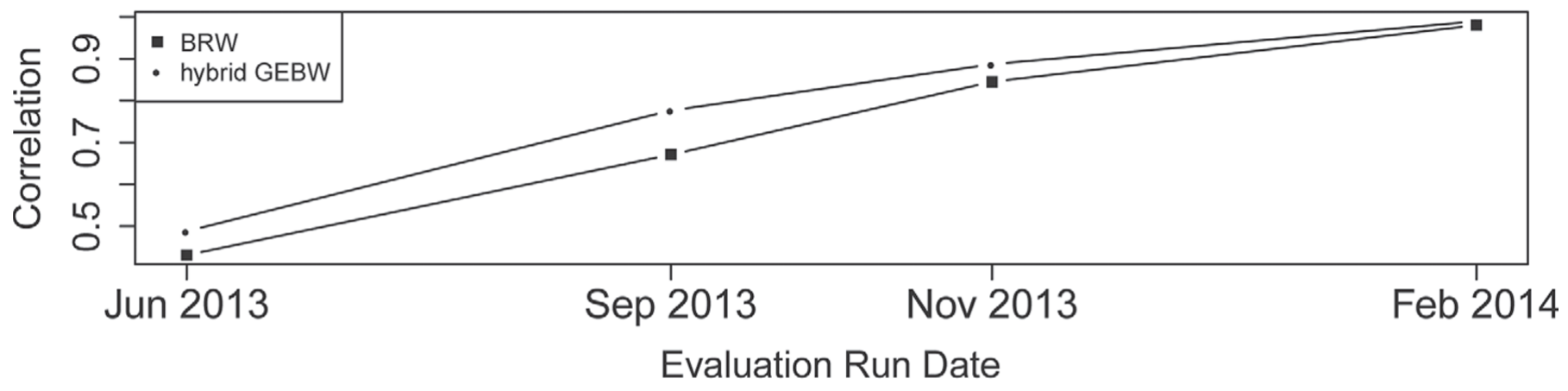

Jerseys

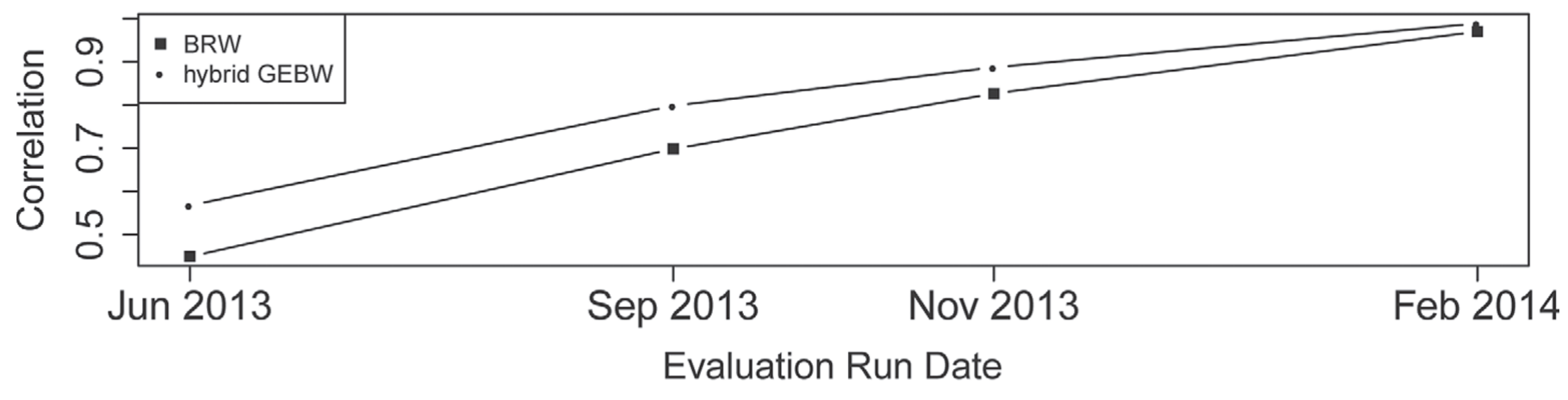

FJ Crosses

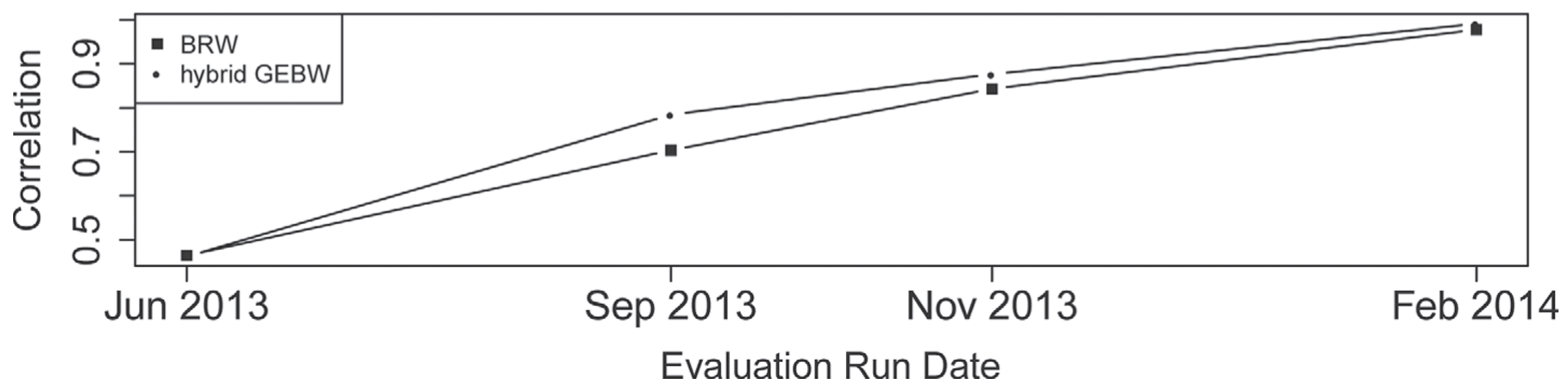

Figure 7. Correlation between the breeding worth (BRW) and genomically estimated BRW (GEBW) calculated in June through February with the April (end-of-season) GEBW. FJ = Friesian-Jersey.

liabilities for BRW and hybrid GEBW. Results from sires with 100 or fewer daughters were included in the graphs (n = 56 Friesians, 33 Jerseys, and 32 FJ crosses). The BRW and GEBW include breeding value and GEBV on residual survival and fertility, which have low heritabilities (5.5 and $2.2 \%$, respectively) and no daughter information from first-lactation daughters. Milk volume, conversely, has a heritability of $34 \%$ and daughter records contribute to the reliability of evaluation starting from the first herd test. Hence, greater variation was noted in BRW and GEBW than in milk breeding value and GEBV. In NZ, bimonthly herd testing is the norm, so heifers calving in July have their first herd test occurring in September. Evaluations calculated in June did not include information from daughter records. The September evaluation included 
data on 1 herd test and the November evaluation included between 1 and 2 herd tests. As expected, the difference between the reliabilities was greatest in June and decreased thereafter. The average difference between the reliabilities of the hybrid GEBW and BRW was 23.0, 10.3, 5.1, 2.8, and 2.6 in June, September, November, February, and April, respectively.

Figure 7 contains the correlations of the BRW and hybrid GEBW calculated in June through February with the GEBW calculated in April 2014. Prior to the inclusion of daughter information, the GEBW were more closely correlated with April GEBW than were the BRW for Friesians and Jerseys, but not the FJ crosses. The difference between the BRW and GEBW decreased throughout the season. By February 2014, the correlations were $97 \%$ or higher for all breeds.

\section{DISCUSSION}

The hybrid method is a computationally feasible method of calculating GEBV. The main benefit was for evaluation of young sires, before the availability of adequate progeny information; selecting unproven sires on GEBW would be more efficient than selecting on BRW. Whereas the hybrid system is feasible at the moment, it does have limitations. Solving the system relies on direct inversion of some matrices (EDM and $\mathbf{A}_{22}$ ). Unless indirect methods of obtaining the inverses are devised, the hybrid system has a limited lifespan if the number of genotyped animals continues to increase. An alternative would be to exclude older genotyped cows, thereby limiting the size of the matrices. The effect of excluding these animals from the hybrid analysis has yet to be determined.

Additional research on the parameters used may result in an increase in accuracy of the genomic evaluations. The system currently uses 3 parameters, bandwidth, mixing, and scaling (equations A1, A2, and A3 in the Appendix, respectively), the first 2 of which were set to constant values for all analyses whereas the scaling parameter was varied. The advantage of having both a scaling and mixing parameter is that, with a constant mixing parameter, different scaling parameters can be assessed without having to invert the $\mathbf{G}_{\theta}$ (the blended genomic relationship matrix) and $\mathbf{A}_{22}$ matrices for each run. We used an experimental approach to find good, but possibly not optimal, values of the scaling parameter for each trait. These would be good starting values for other researchers, but a numerical approach, if computationally practical, could result in more optimal values. The bandwidth controls rate of the decay of the correlation; the larger the $\mathrm{h}$, the stronger the correlation. The effect of changing the magnitude of $\mathrm{h}$ on the resulting evaluations was not assessed in the present study. Preliminary work on varying this parameter showed that changing the bandwidth affected the inflation but not the accuracy of the GEBV. In the present study, the inflation was controlled using the scaling parameter and little would be gained by also adjusting the bandwidth. The GRM can be formed in different ways; VanRaden (2008) and Forni et al. (2011) use a cross-product of allele counts scaled in different ways (for example, equation A4 in the Appendix). The EDM was used in the present study for computational reasons; we were not able to obtain convergence when solving the equations in which the GRM was used. The EDM and GRM have different properties; the EDM is a distance measure that ranges from greater than 0 and 1. The $\mathbf{G}$ matrix can have values outside this range. The ways and degrees to which the choice of matrix affects the resulting GEBV have not been fully studied and requires further consideration.

Weighting of SNP that were more closely related to traits of interest than other SNP provided little improvement in the accuracy of the genomic evaluations. The SNP were from a $50 \mathrm{~K}$ panel that may not have been dense enough to capture the QTL. Repeating the analyses with high-density $(\approx 800 \mathrm{~K})$ genotypes or SNP derived from sequence data may better capture the causative alleles than in the present study.

\section{CONCLUSIONS}

Our study compared 2 methods of calculating GEBV. The GEBV were calculated for the 7 traits that are in the national breeding objective. The hybrid method that used the augmented pedigree matrix and the DRBV derived from the traditional evaluation was found to produce GEBV that were considerably less inflated and more accurate than GEBV obtained by blending DGV and traditional EBV. The estimates of inflation of the predicted blended GEBV of the test sires ranged from 0.34 to 0.83 ; the range was 0.93 to 1.06 for the hybrid GEBV. The correlations between the traditional EBV and predicted blended GEBV of the test sires ranged from 0.38 to 0.77 . The range for the hybrid GEBV was 0.56 to 0.84 ; the production traits had correlations that averaged 0.07 to 0.12 higher than was found for the blended GEBV. The genomic information was found to improve the predictive ability of unproven bulls by accounting for MS deviations, which are not included in the parent average breeding value. The difference in the reliabilities of the traditional and hybrid EBV and BRW diminished substantially after the second herd test of the first-crop daughters of the young sires. Genomic information had little to contribute to a sire's EBV after the records on the second herd test of firstcrop daughters was included in the EBV estimation. 


\section{REFERENCES}

Aguilar, I., I. Misztal, D. L. Johnson, A. Legarra, S. Tsuruta, and T. J. Lawlor. 2010. Hot topic: A unified approach to utilize phenotypic, full pedigree, and genomic information for genetic evaluation of Holstein final score1. J. Dairy Sci. 93:743-752.

Blott, S., J.-J. Kim, S. Moisio, A. Schmidt-Küntzel, A. Cornet, P. Berzi, N. Cambisano, C. Ford, B. Grisart, D. Johnson, L. Karim, P. Simon, R. Snell, R. Spelman, J. Wong, J. Vilkki, M. Georges, F. Farnir, and W. Coppieters. 2003. Molecular dissection of a quantitative trait locus: A phenylalanine-to-tyrosine substitution in the transmembrane domain of the bovine growth hormone receptor is associated with a major effect on milk yield and composition. Genetics 163:253-266.

Browning, S. R., and B. L. Browning. 2007. Rapid and accurate haplotype phasing and missing-data inference for whole-genome association studies by use of localized haplotype clustering. Am. J. Hum. Genet. 81:1084-1097. http://dx.doi.org/10.1086/521987.

Chamberlain, A. J., B. J. Hayes, K. Savin, S. Bolormaa, H. C. McPartlan, P. J. Bowman, C. Van der Jagt, S. MacEachern, and M. E. Goddard. 2012. Validation of single nucleotide polymorphisms associated with milk production traits in dairy cattle. J. Dairy Sci. 95:864-875.

Fernando, R., and D. Garrick. 2009. User manual for a portfolio of genomic selection related analyses. 2nd ed. Iowa State University, Ames.

Forni, S., I. Aguilar, and I. Misztal. 2011. Different genomic relationship matrices for single-step analysis using phenotypic, pedigree and genomic information. Genet. Sel. Evol. 43:1. http://dx.doi. org/10.1186/1297-9686-43-1.

Gianola, D., and J. B. C. H. M. van Kaam. 2008. Reproducing kernel Hilbert spaces regression methods for genomic assisted prediction of quantitative traits. Genetics 178:2289-2303.

Habier, D., R. L. Fernando, and J. C. M. Dekkers. 2007. The impact of genetic relationship information on genome-assisted breeding. Genetics 177:2389-2397.

Habier, D, R. L. Fernando, K. Kizilkaya, and D. J. Garrick. 2011. Extension of the Bayesian alphabet for genomic selection. Bioinformatics 12:186-198.

Harris, B. L., D. L. Johnson, and R. J. Spelman. 2011. Choice of parameters for removal of inflation in genomic breeding values for dairy cattle. Pages 359-362 in Proc. Assoc. Adv. Anim. Breed. Genet., vol. 19. Association for the Advancement of Animal Breeding and Genetics, Perth, Australia.

Harris, B., and D. L. Johnson. 1998. Approximate reliability of genetic evaluations under an animal model. J. Dairy Sci. 81:2723-2728. http://dx.doi.org/10.3168/jds.S0022-0302(98)75829-1.

Harris, B. L., and D. L. Johnson. 2010. Genomic predictions for New Zealand dairy bulls and integration with national genetic evaluation. J. Dairy Sci. 93:1243-1252. http://dx.doi.org/10.3168/ jds.2009-2619.

Harris, B. L., A. M. Winkelman, and D. L. Johnson. 2012. Large-scale single-step genomic evaluation for milk production traits. Interbull Bull. 46:20-24.

Johnson, D. L. 1996. Estimation of lactation yield from repeated measures of test day yields. Proc. N.Z. Soc. Anim. Prod. 56:16-18.

Karim, L., H. Takeda, L. Lin, T. Druet, J. A. C. Arias, D. Baurain, N. Cambisano, S. R. Davis, F. Farnir, B. Grisart, B. L. Harris, M. D. Keehan, M. D. Littlejohn, R. J. Spelman, M. Georges, and W. Coppieters. 2011. Variants modulating the expression of a chromosome domain encompassing PLAG1 influence bovine stature. Nat. Genet. 43:405-413. http://dx.doi.org/10.1038/ng.814.

Legarra, A., I. Aguilar, and I. Misztal. 2009. A relationship matrix including full pedigree and genomic information. J. Dairy Sci. 92:4656-4663.

Legarra, A., and V. Ducrocq. 2012. Computational strategies for national integration of phenotypic, genomic, and pedigree data in a single-step best linear unbiased prediction. J. Dairy Sci. 95:46294645. http://dx.doi.org/10.3168/jds.2011-4982.

Makgahlela, M. L., I. Strandén, U. S. Nielsen, M. J. Sillanpää, and E. A. Mäntysaari. 2013. The estimation of genomic relationships using breedwise allele frequencies among animals in multibreed populations. J. Dairy Sci. 96:5364-5375.

Meredith, B. K., D. P. Berry, F. Kearney, E. K. Finlay, A. G. Fahey, D. G. Bradley, and D. J. Lynn. 2013. A genome-wide association study for somatic cell score using the Illumina high-density bovine beadchip identifies several novel QTL potentially related to mastitis susceptibility . Front. Genet. 4:229.

Meuwissen, T. H. E., B. J. Hayes, and M. E. Goddard. 2001. Prediction of total genetic value using genome-wide dense marker maps. Genetics 157:1819-1829.

Minozzi, G., E.L. Nicolazzi, A. Stella, S. Biffani, R. Negrini, B. Lazzari, P. Ajmmone-Marsan, and J. L. Williams. 2013. Genome wide analysis of fertility and production traits in Italian Holstein cattle. PLoS ONE 8:e80219.

Misztal, I., A. Legarra, and I. Aguilar. 2009. Computing procedures for genetic evaluation including phenotypic, full pedigree, and genomic information. J. Dairy Sci. 92:4648-4655. http://dx.doi. org/10.3168/jds.2009-2064.

Misztal, I., Z. G. Vitezica, A. Legarra, I. Aguilar, and A. A. Swan. 2013. Unknown-parent groups in single-step genomic evaluation. J. Anim. Breed. Genet. 130:252-258. http://dx.doi.org/10.1111/ jbg. 12025 .

Pryce, J. E., B. J. Hayes, S. Bolormaa, and M. E. Goddard. 2011. Polymorphic regions affecting human height also control stature in cattle. Genetics 187:981-984. http://dx.doi.org/10.1534/genetics.110.123943.

Quaas, R. L. 1988. Additive genetic model with groups and relationships. J. Dairy Sci. 71:1338-1345.

Raven, L.-A., B. G. Cocks, and B. J. Hayes. 2014. Multibreed genome wide association can improve precision of mapping causative variants underlying milk production in dairy cattle. BMC Genomics 15:62-76.

Sahana, G., B. Guldbrandtsen, B. Thomsen, and M. S. Lund. 2013. Confirmation and fine-mapping of clinical mastitis and somatic cell score QTL in Nordic Holstein cattle. Anim. Genet. 44:620-626.

Spelman, R. J., C. A. Ford, P. McElhinney, G. C. Gregory, and R. G. Snell. 2002. Characterization of the DGAT1 gene in the New Zealand dairy population. J. Dairy Sci. 85:3514-3517. http://dx.doi. org/10.3168/jds.S0022-0302(02)74440-8

Strandén, I., and M. Lidauer. 1999. Solving large mixed linear models using preconditioned conjugate gradient iteration. J. Dairy Sci. 82:2779-2787. http://dx.doi.org/10.3168/jds.S00220302(99) 75535-9.

Su, G., P. Madsen, U. S. Nielsen, E. A. Mäntysaari, G. P. Aamand, O. F. Christensen, and M. S. Lund. 2012. Genomic prediction for Nordic Red Cattle using one-step and selection index blending. J Dairy Sci. 95:909-917. http://dx.doi.org/10.3168/jds.2011-4804.

VanRaden, P. M. 2008. J. Efficient methods to compute genomic predictions. J. Dairy Sci. 91:4414-4423.

Vitezica, Z. G., I. Aguilar, I. Misztal, and A. Legarra. 2011. Bias in genomic predictions for populations under selection. Genet. Res. (Camb.) 93:357-366. http://dx.doi.org/10.1017/ S001667231100022X.

Wiggans, G. R., T. S. Sonstegard, P. M. VanRaden, L. K. Matukumalli, R. D. Schnabel, J. F. Taylor, F. S. Schenkel, and C. P. Van Tassell. 2009. Selection of single-nucleotide polymorphisms and quality of genotypes used in genomic evaluation of dairy cattle in the United States and Canada. J. Dairy Sci. 92:3431-3436. http://dx.doi.org/10.3168/jds.2008-1758.

\section{APPENDIX}

\section{Genomic Relationships}

Let $\mathbf{M}$ denote the $n \times q$ matrix of genotypes (coded $0,1,2$ ) for $n$ genotyped animals and $q$ SNP markers. The matrix $\mathbf{G}$ is based on Euclidean distance in a Gaussian kernel (EDM) 


$$
\mathbf{G}=\exp (-\overline{\mathbf{E}} / h)
$$

where

$$
\mathbf{E}_{i j}=\sum_{k}\left(m_{i k}-m_{j k}\right)^{2}
$$

for the $k$ th marker $(m)$ of individuals $i$ and $j$,

$$
\begin{gathered}
\overline{\mathbf{E}}_{i j}=\frac{\mathbf{E}_{i j}}{u_{m}} \\
u_{m}=\frac{\sum_{i}^{n} \sum_{j}^{n} \mathbf{E}_{i j}}{n^{2}}
\end{gathered}
$$

and $h$ is a bandwidth parameter (Gianola and van Kaam, 2008) that was set to 1.333 for all analyses.

The matrix $\mathbf{E}$ can be conveniently derived using

$$
\mathbf{E}_{i j}=\mathbf{S}_{i}+\mathbf{S}_{j}-2 \mathbf{M M}_{i j}^{\prime}
$$

where

$$
\mathbf{S}=\operatorname{Diag}\left[\mathbf{M M}^{\prime}\right]
$$

The genomic relationships can be blended with the corresponding pedigree-based matrix $\mathbf{A}_{22}$ to yield

$$
\mathbf{G}_{\theta}=\theta \mathbf{G}+(1-\theta) \mathbf{A}_{22},
$$

where $\theta$ is the mixing parameter [initially described by VanRaden (2008)] set to 0.80 for all analyses.

The inverse of the relationship matrix combining pedigree and genomics for all animals is then given by

$$
\mathbf{H}^{-1}=\mathbf{A}^{-1}+\left[\begin{array}{lc}
0 & 0 \\
0 & \varphi\left(\mathbf{G}_{\theta}^{-1}-\mathbf{A}_{22}^{-1}\right)
\end{array}\right],
$$

which involves the mixing parameter $\theta$ and scaling parameter $\varphi$. The role of $\varphi$ in weighting different sources of information (parent average, genomic prediction, and subset pedigree prediction) in genetic evaluation is shown in Appendix B of Aguilar et al. (2010).

In the previous derivation of $\mathbf{E}$, the summation over SNP can be weighted in proportion to SNP-specific variances, if desired, to give due recognition to regions of the genome with a large effect on the trait, for example, the effect of DGAT on production traits.

Alternatively, a GRM can be calculated as (Habier et al., 2007)

$$
\mathbf{G}=\frac{\mathbf{M M}^{\prime}-4 \mathbf{P P}^{\prime}}{2 \sum_{m} p_{m}\left(1-p_{m}\right)},
$$

where $\mathbf{P}_{i m}=p_{m}$, and $p_{m}$ is allele frequency for SNP $m$. For a multibreed population, a regression procedure was proposed to account for differences in allele frequencies among breeds (Harris and Johnson, 2010). However, this method is computationally demanding for the number of genotyped animals considered here. A simpler breed adjustment is to define

$$
\mathbf{P}=\sum_{k} \lambda_{i k} p_{k m}
$$

where $\lambda_{i k}$ is the fraction of breed $k$ in animal $i$ and $p_{k m}$ is allele frequency for breed $k$ at SNP $m$. The breed-specific allele frequencies can be estimated from Holstein-Friesian and Jersey bulls using the approach of Makgahlela et al. (2013). Then, to correct for selection (Vitezica et. al., 2011), the matrix is adjusted as

$$
\mathbf{G}^{\S}=(1.0-0.5 \alpha) \mathbf{G}+\alpha \mathbf{1 1},
$$

where $\pm=\frac{1}{n} \sum_{i j}(\mathbf{A}-\mathbf{G})_{i j}$ and, similar to $\mathbf{E D M}, \mathbf{G}$ can be blended with $\mathbf{A}_{22}$.

\section{EDM Incorporating SNP Variances}

$\mathbf{M}$ is the $n \times q$ matrix of genotypes and $\mathbf{w}$ is the vector of SNP variances. Then the weighted distance matrix has elements

$$
\mathbf{E}_{i j}=\mathbf{S}_{i}+\mathbf{S}_{j}-2 \mathbf{M W} \mathbf{M}_{i j}^{\prime}
$$

where

$$
\mathbf{S}=\operatorname{Diag}\left[\mathbf{M W M}^{\prime}\right]
$$

and $\mathbf{W}$ is the diagonal matrix containing the weights, w. 